\title{
Modelling the Temperature Evolution During Hot Reversing Strip Rolling of Magnesium Alloys
}

\author{
Alexander, Nam ${ }^{1, \mathrm{a}}$, Uwe, Prüfert ${ }^{2, \mathrm{~b}}$, \\ Michael, Eiermann ${ }^{2, \mathrm{c}}$, and Rudolf, Kawalla ${ }^{1, \mathrm{~d}}$ \\ ${ }^{1}$ Institute of Metal Forming, TU Bergakademie Freiberg, \\ Bernhard von Cotta Straße 4, 09599 Freiberg, Germany \\ ${ }^{2}$ Institute of Numerical Analysis and Optimization, TU Bergakademie Freiberg, \\ Akademiestraße 9, 09599 Freiberg, Germany \\ aalexander.nam@imf.tu-freiberg.de, buwe.pruefert@math.tu-freiberg.de, \\ cmichael.eiermann@math.tu-freiberg.de, ${ }^{\mathrm{d}}$ rudolf.kawalla@imf.tu-freiberg.de
}

Keywords: Reversing rolling; thermal modelling; 3-D simulation; magnesium alloys

\begin{abstract}
The paper proposes the approach for the thermal through process modelling of the strip hot rolling chain of magnesium alloys. This strip hot rolling chain comprises the coil reheating after the Twin-Roll Casting (TRC) process, storage or transport operations and reversing hot rolling. The modelling of reversing rolling is implemented in connection with the simultaneous un-/coiling process of a coil. The numerical calculation is based on an object-oriented FEM tool kit written in MATLAB ${ }^{\text {TM }}$ and is carried out in three spatial dimensions and time.
\end{abstract}

\section{Introduction}

Magnesium alloy strips are being increasingly used in electronics, the automotive and aerospace industries due to their low density, high specific strength, good damping capacity and outstanding thermal diffusivity, etc. However, being one of the hexagonal close-packed crystal structure metals, magnesium alloys exhibit poor plasticity at low temperatures. Therefore, magnesium alloy strips are generally rolled at elevated temperatures. One of the innovative and energy-saving technological process chains for the production of magnesium alloy strips is the combined technology of twin roll casting (TRC) and reversing hot rolling, which has been developed by the Institute of Metal Forming at the TU Bergakademie Freiberg in collaboration with the MgF Magnesium Flachprodukte GmbH Freiberg [1].

Twin roll casting

Heat treatment

Strip rolling

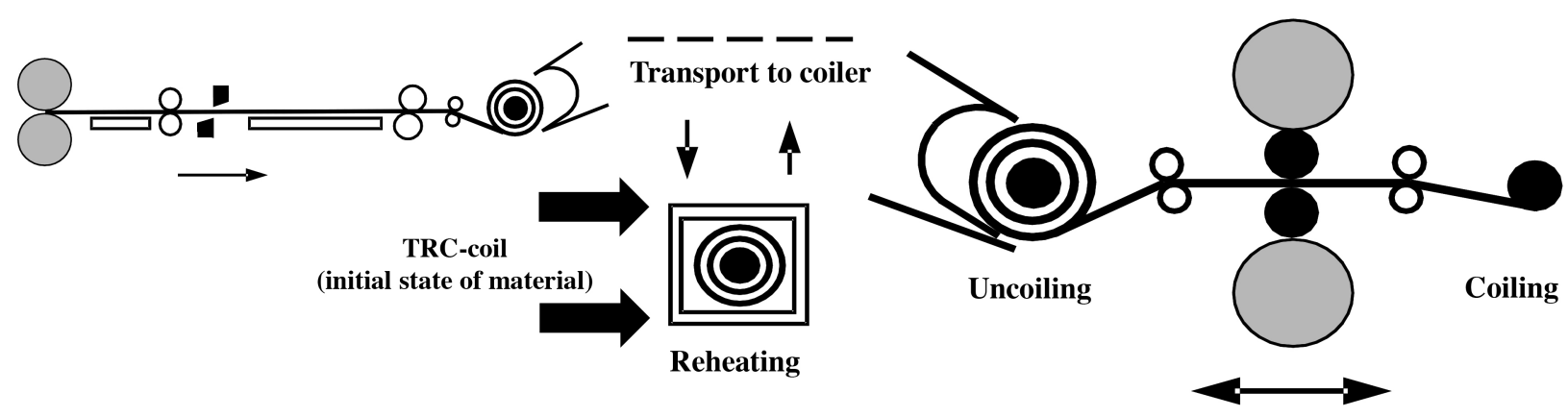

Fig. 1: Technological chain of magnesium strip rolling

The paper focuses on the thermal modelling of the reversing strip rolling of magnesium alloy strip in connection with the un-/coiling process of the coils. Moreover, this modelling approach is connected 
with previous steps such as reheating and storage or the transport operation. The modelling of previous steps and their results are described in $[2,3]$.

The presented work proposes a modelling approach for the calculation of the thermal evolution during the reversing rolling process of magnesium alloys, taking into account the un-/coiling process of coils. The current state of work on this modelling approach is given and the results will be presented and discussed in conclusion.

\section{Finite Element Formulation}

Define $\Omega \subset R^{3}$ as a bounded domain, which is divided in sub-domains $\Omega_{c 1}, \Omega_{c 2}$ and $\Omega_{s}$. Index $c 1$ or $c 2$ denotes the coil area of coil1 or coil2 and index $s$ stands for the unrolled strip. $\Gamma_{l}, l=1, \ldots, n_{b s}$ are the $n_{b s}$ surface segments of $\Omega$. These segments are used to define several boundary conditions modelling contact with surrounding air, a mandrel, or guide pulleys. During the processing steps the form of $\Omega$ as well as the number of boundary conditions varies. The convective-diffusion equation for modelling heat transfer cf. [4], is written as

$$
\begin{aligned}
c_{p} \rho \frac{\partial T}{\partial t} & =\nabla^{T} k \nabla T+F & & \text { on } \Omega, \\
n \cdot(k \nabla T) & =-h_{l}\left(T-T_{\Gamma_{l}}\right) & & \text { on } \Gamma_{l}, \quad l=1, \ldots, n_{b s}
\end{aligned}
$$

where $\mathrm{T}(\mathrm{K})$ is the temperature, $\rho\left(\mathrm{kg} / \mathrm{m}^{3}\right)$ is the density, $\mathrm{c}_{p}(\mathrm{~J} /(\mathrm{kg} \mathrm{K}))$ is the specific heat capacity and $\mathrm{h}_{l}\left(\mathrm{~W} /\left(\mathrm{m}^{2} \mathrm{~K}\right)\right)$ is the heat transfer coefficient according to the boundary conditions. In $\Omega_{c}$ the matrix $k=\left\{k_{i, j}\right\}_{i j=1, \ldots, 3}(\mathrm{~W} /(\mathrm{m} \mathrm{K}))$ contains the thermal conductivity according to the spatial directions. The initial temperature is given by $T\left(t_{0}\right)=T_{\text {start }}$. A radiative heat transfer boundary condition is not considered in the presented model in order to simplify and to accelerate the calculation. However, the emissivity of magnesium alloys in the temperature range of the reversing rolling of magnesium alloys is about 0.135 . The radiation of magnesium alloy will be taken into account in the further development of the work. To apply the finite element method (FEM) we consider the weak form of the differential equation. We obtain the variational equation

$$
\int_{\Omega} \rho c_{p} \frac{\partial T(t, x)}{\partial t} v(x) d x=\int_{\Omega} \nabla^{T} k(T(t, x)) \nabla T(t, x) v(x) d x+\int_{\Omega} f v(x) d x \text { for all } v \in V,
$$

cf. the classical textbook [5]. After applying Green's formula to Eq. 3 and inserting the boundary conditions we obtain

$$
\begin{aligned}
\int_{\Omega} \rho c_{p} \frac{\partial T(t, x)}{\partial t} v(x) d x=-\sum_{l=1}^{n_{b s}} \int_{\Gamma_{l}} h_{l}(T(t, x) & \left.-T_{\Gamma_{l}}(t)\right) v(x) d s+ \\
& +\int_{\Omega} k(T(t, x)) \nabla T(t, x) \nabla v(x) d x+\int_{\Omega} f v(x) d x
\end{aligned}
$$

After discretization of the domain $\Omega$, boundary $\Gamma$, and of all functions by finite elements, we finally obtain the semi-discrete problem

$$
D \frac{\partial T}{\partial t}+\left(K(T)+\sum_{l=1}^{n_{b s}} H_{l}\right) T=\sum_{l=1}^{n_{b s}} G_{l}+F
$$

where $\mathrm{K}$ is the stiffness matrix, $\mathrm{D}$ is the mass matrix, $\mathrm{F}$ is the vector of the heat source and the matrices $H_{l}$ correspond to functions $h_{l}$ and $G_{l}$. They are the vectors corresponding to the given data $T_{\Gamma_{l}}$ in the boundary integrals. By the dependence of the stiffness matrix on the temperature this is a non-linear problem. For the details of the used FEM software OOPDE we refer to [6]. 


\section{Thermal Modelling of Reversing Rolling}

The idea of the modelling approach consists of the performing of the thermal modelling of the reversing rolling process in between two models of the coils, which are synchronously uncoiled on the one side and coiled on the opposite side. For this purpose a coil model and the coiling process of coil have been worked out and have been connected with each other.

Coil Geometry. The heat transfer problem is considered in the Cartesian coordinates instead of the more usual the cylindrical coordinates $[7,8,9,10]$. However, the $3-\mathrm{D}$ domain $\Omega$ is still a cylinder in the formal mathematical sense, i.e. we can construct $\Omega$ by extruding a 2 -D object. This 2 -D object has an outer boundary given by the curve $c_{\text {out }}$ and an inner boundary $c_{i n}, s \in[0,2 \pi]$ in case of rolled coil, see Eq. $6-7$.

$$
\begin{aligned}
c_{\text {out }}(s) & =\left(\begin{array}{c}
R_{\text {out }}(s) \sin (s) \\
-R_{\text {out }}(s) \cos (s)
\end{array}\right) & c_{\text {in }}(s) & =\left(\begin{array}{c}
-R_{\text {in }}(s) \sin (s) \\
R_{\text {in }}(s) \cos (s)
\end{array}\right) \\
R_{\text {out }}(s) & =\left(r_{\text {out }}-d_{\text {sheet }}\right) \frac{s-2 \pi}{2 \pi}+r_{\text {out }} \frac{s}{2 \pi} & R_{\text {in }}(s) & =\left(r_{\text {in }}+d_{\text {sheet }}\right) \frac{s-2 \pi}{2 \pi}+r_{\text {in }} \frac{s}{2 \pi}
\end{aligned}
$$

This is the exact coil geometry depending on the outer radius. The inner radius is pre-determined and given by the diameter of the mandrel and the thickness of the magnesium strip $d_{\text {sheet }}$.

Modelling of the Un- and Coiling Process. By modelling of un-/coiling process the coil is considered in connection with the un-/coiled strip as an entirety. Moreover, the volume of the coil and the un-/coiled strip must be constant. It means that the outer diameter of the unrolled coil decreases whereas the length of the uncoiled strip increases per time unit. In case of the coiling process it occurs inversely. The major complexity of this modelling is the data transport after the modification of the geometry.
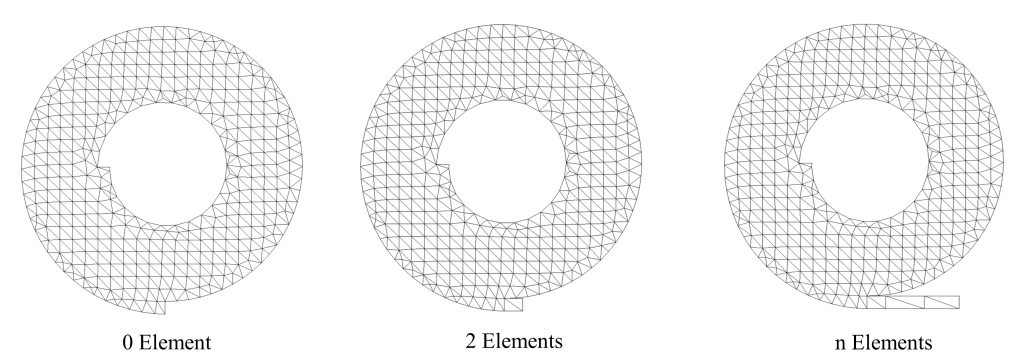

Fig. 2: Algorithm to the uncoiling process by adding of elements

The geometrical modelling of the un-/coiling process is performed using the advantage of the geometry of the coil model. The uncoiling process is implemented by means of adding elements to the strip tail end of coil. Moreover, as soon as the length of the unrolled strip reaches the circumference of the outer diameter of the coil, the coil will shrink.

The processes of element addition and coil shrinking are repeated until the length of the unrolled strip achieves the position of the second coil. When the length of the unrolled strip from the position of the second coil amounts to the diameter of the mandrel, the second coil model will be created. In the next steps the adding of elements will be continued in connection with the second coil. As soon as the length of the unrolled strip achieves the outer diameter of the second coil, the volume of the second coil will be increased by the respective circumference of the next outer diameter. It should be noted that the strip will not be unrolled completely by the uncoiling process. At least three windings always remain on the mandrel. This reversing rolling can also be performed in the opposite direction. In this case the second coil will be uncoiled while the first coil will be coiled.

The idea of the data transport during reversing rolling consists of the splitting of the rolling process into parts: coil1, strip1 (strip before the roll gap), strip2 (strip after the roll gap) and coil2. In each part the data is locally interpolated and subsequently the interpolated parts are further connected with each 
other by means of global interpolation. Moreover, the data is transported along these parts per time step. So the data is moved by the uncoiling process from the coil into the unrolled strip and conversely by the coiling process.

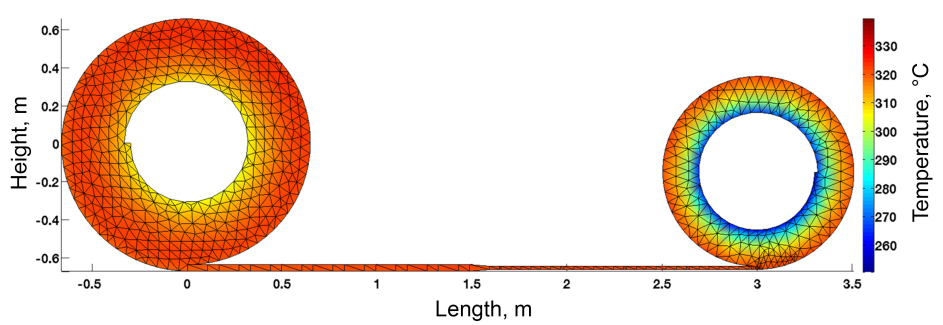

Fig. 3: Example of calculated units of reversing rolling by means of interpolation

Calculation of Dissipated Heat in Roll Gap. The roll gap is modelled under the assumption that the deformation in the roll gap occurs rigid-plastically. In this case the dissipated heat is related to the average deformation degree within the entire volume of the roll gap. The dissipated heat through plastic deformation can be calculated by means of the following equation:

$$
\dot{Q}=\eta \bar{\sigma} \dot{\bar{\varepsilon}}, \quad \eta=0.9-0.95
$$

In Eq. 8, $\dot{\mathrm{Q}}$ is the rate of deformation heat and equals zero at the various thermal zones, except for the deformation zone. $\bar{\sigma}$ and $\dot{\bar{\varepsilon}}$ are the effective stress and effective strain rate, respectively, see [11]. In view of the FEM this dissipated heat through plastic deformation will be taken into account in the middle of every element inside the roll gap area.

The contact of the strip with the rolls is performed through the convective boundary conditions, as stated previously.

\section{Results of Simulation}

The implementation of the models has been carried out in MATLAB by using the object-oriented FEM Toolbox OOPDE [6]. The software is modularized, i.e. for every step in the technological chain $[1,3]$ there is a separated class. These classes are derived from an abstract super-class that implements all common properties and methods such as e.g. non-linear solvers, time integrators etc., see [6] for details.

Table 1 shows the applied parameters by FEM calculation of the reversing rolling.

Table 1: Finite element analysis conditions for the heating/cooling simulation

\begin{tabular}{lll} 
Magnesium & Element type for discretization & Prism \\
coil / strip & Specific heat capacity $/\left[\mathrm{J} \cdot \mathrm{kg}^{-1} \cdot \mathrm{C}^{-1}\right]$ & $1054.7 \cdot \mathrm{e}^{0.0005 \cdot T}$ \\
& Density $/\left[\mathrm{kg} \cdot \mathrm{m}^{-3}\right]$ & 1738 \\
Heat transfer & contact with rolls & $0.5 \cdot 100.2 \cdot e^{3.06+1.57 \cdot 0.003 \cdot T_{\text {rolls }}}$ \\
coefficient in & within coil, i.e. equivalent radial conductivity & $19.72 \cdot\left(1-e^{-(T / 131.79)^{0.89}}\right)$ \\
$\frac{W}{\mathrm{~m}^{2} \cdot \mathrm{K}}$ & in ambient air, i.e. free surface & $3.7535 \cdot T^{0.236}$ \\
& contact with pulley guide & 52 \\
\hline
\end{tabular}

Fig. 4 demonstrates some calculated steps of reversing rolling. The dimensions of the coil and the strip can be taken from Fig. 4. The rolling speed is $0.5 \mathrm{~m} / \mathrm{s}$. Fig. 4(a) shows the temperature state of the reversing rolling with the uncoiling of the first coil. It can be noticed that the temperature drop from the beginning of the coil uncoiling to the centre of the second coil is about $10{ }^{\circ} \mathrm{C}$. In Fig. 4 (b) and 4(c) the second coil has already been created. The rolling process is carried out with both the 
uncoiling and coiling process. The temperature evolution and the difference between both coils can be observed. Fig. 4(d) exhibits the completely uncoiled first coil and the coiled second coil, respectively. As it has already been mentioned above the completely uncoiled coil comprises at least three windings. Moreover, it can be noticed that the temperature state of the second coil after the first rolling pass with set rolling parameters is about $320^{\circ} \mathrm{C}$. The temperature reduction is approx. $50{ }^{\circ} \mathrm{C}$.

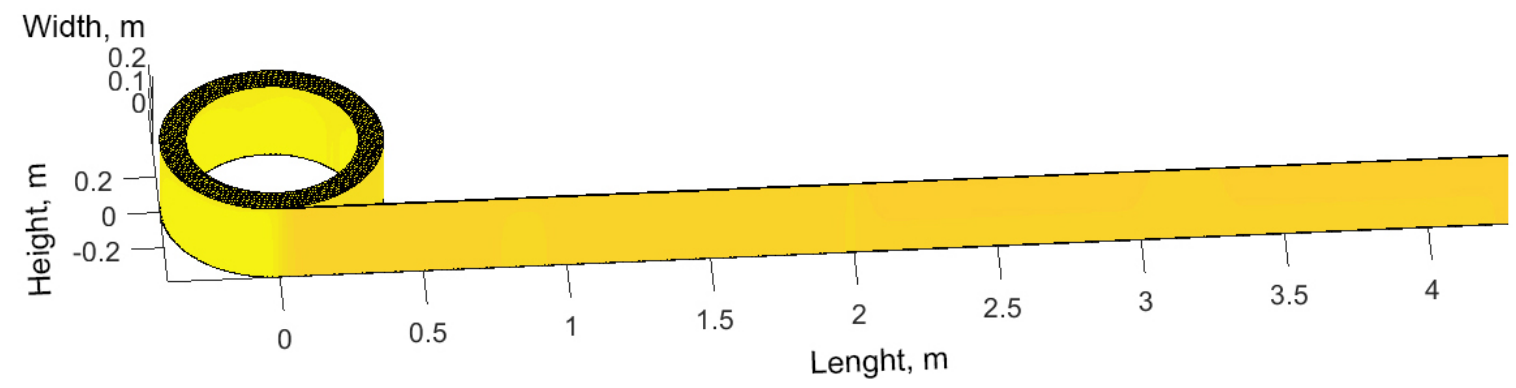

(a) Reversing rolling with only unrolling process of coill

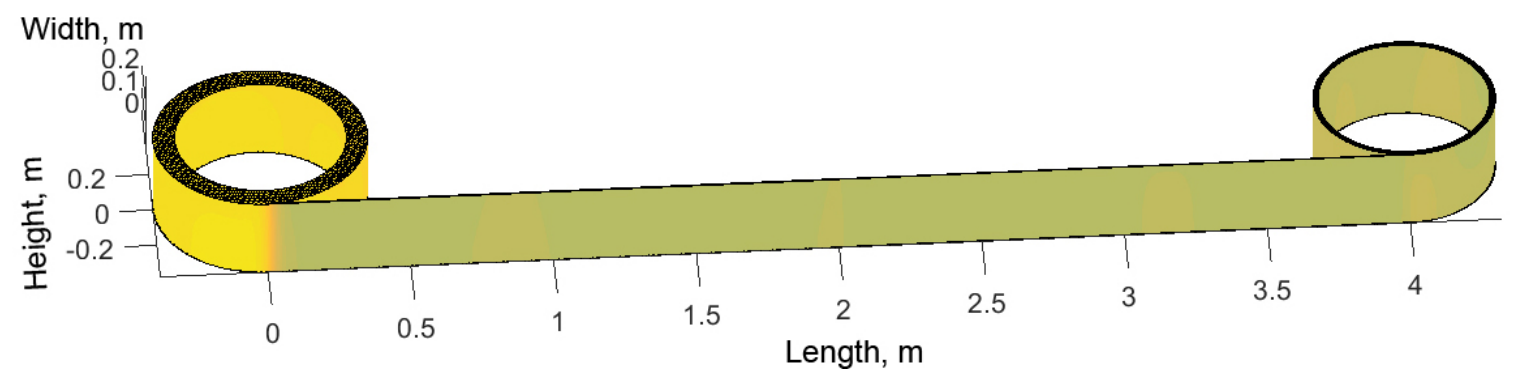

(b) Reversing rolling with unrolling process of coill and begin of coiling process of coil2

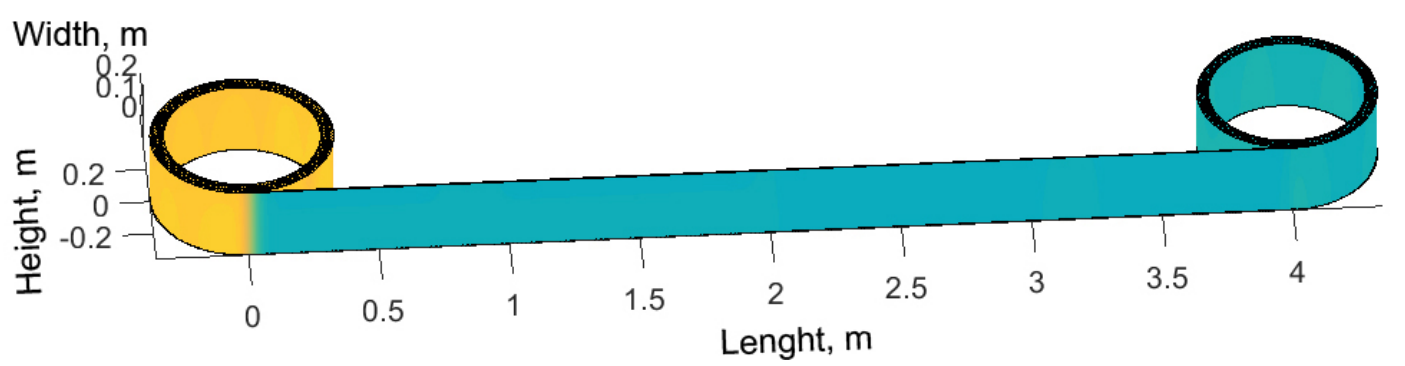

(c) Continuation of reversing rolling

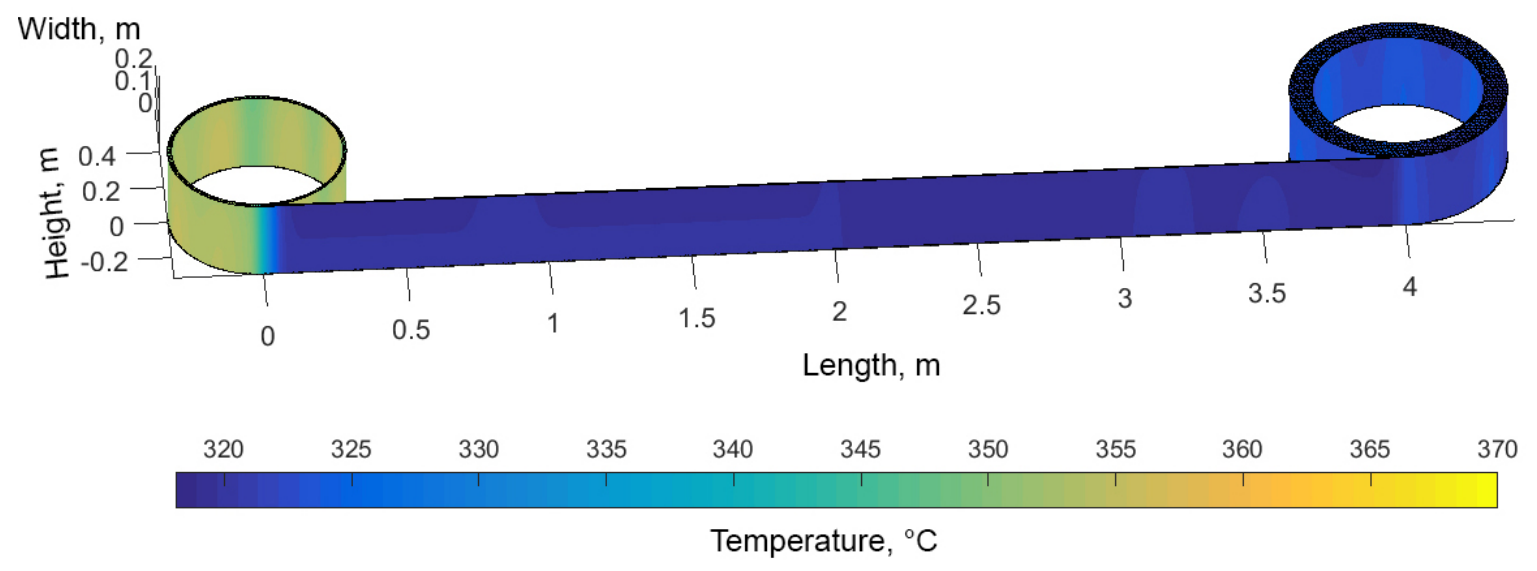

(d) Reversing rolling with complete uncoiled coil1 and coiled coil2, respectively

Fig. 4: Demonstration of modelling of reversing rolling step by step

In the near future experimental trials will be planned to determine the missing parameters on the one hand, and to validate the simulation results with experimental ones, on the other hand. 


\section{Summary}

The paper focuses on the thermal modelling of reversing rolling of magnesium alloys. The proposed modelling approach enables the analysis of the temperature state of reversing rolling taking into account the un-/coiling process of a coil. The first calculations have been implemented and discussed. In the near future experimental trials will be carried out in order to validate the simulation results on the one hand, and to determine the missing parameters, on the other hand. The advantage of this modelling approach consists on the possibility to calculate both the temperature state of coils and the temperature state of the unrolled strip in connection with coils. Based on these calculations the microstructure evolution can be calculated along the reversing rolling process more accurately.

\section{References}

[1] R. Kawalla, M. Ullmann, M. Oswald, Chr. Schmidt, Properties of strips and sheets of magnesium alloy produced by casting-rolling technology, in: Proceedings of the 7th International Conference on Magnesium Alloys and Their Applications, edited by KU Kainer, Wiley-VCH, (2006)

[2] A. Nam, U. Prüfert, M. Eiermann and R. Kawalla, Concept of thermal modelling for hot strip rolling of magnesium: submitted to Computer Methods in Material Science (2015), p.29

[3] A. Nam, U. Prüfert, M. Eiermann and R. Kawalla, Numerical Modeling of Thermal Evolution in Hot Strip Rolling of Magnesium Alloy, Key Engineering Materials, Vol.651-653, (2015), p.207

[4] M.N. Ozisik, in Heat conduction, Wiley-VCH,(1993).

[5] O.C. Zienkiewicz, and Y. K. Cheung. The Finite Element Method in Structural and Continuum Mechanics. McGraw Hill (1967).

[6] U. Prüfert, OOPDE - An object oriented approach to finite elements in MATLAB, Quickstart Guide (2014), on http://www.mathe.tu-freiberg.de/nmo/mitarbeiter/uwe-pruefert/software.

[7] S.J. Park and B.H. Hong, Finite element analysis of hot rolled coil cooling, ISIJ international Vol.38, No.11 (1998), p.1262.

[8] S.C. Baik, O. Kwon, S.J. Park, B.H. Hong and K.H. Oh, Analysis of heat transfer in hot rolled coils for optimum condition of forced cooling, Metals and Materials Vol.5, No.4 (1999), p.369.

[9] N. Troyani, Nonlinear geometrically adaptive finite element model of the coilbox, Numerical Heat Transfer, Part A Applications (1996), p. 849.

[10] N. Troyani and L. Montano, A temperature predicting model for manufacturing processes requiring coiling, Jo. of the Braz. Soc. of Mech. Scien. Vol.21, No.4 (1999), p. 655.

[11] M. Ullmann, M. Schmidtchen and R. Kawalla, Dynamic recrystallization behaviour of Twin Roll Cast AZ31 strips during hot deformation, Key Engineering Materials, Vol.622, (2014), p.569 